\title{
Dosimetric comparison among different head and neck radiotherapy techniques using PRESAGE® dosimeter
}

\author{
Jalil Rehman ${ }^{1,2,3}$, Tahir Iqbal1', Ramesh Tailor ${ }^{3}$, Abdul Majid ${ }^{1}$, Jahenzeb Ashraf ${ }^{2}$, Isa Khan ${ }^{4}$, \\ Muhammad Afzal', Geoffrrey Ibbott ${ }^{3}$ \\ ${ }^{I}$ Department of Physics, Faculty of Science, University of Gujrat, Hafiz Hayat Campus, Gujrat, Pakistan \\ ${ }^{2}$ Department of Physics, The Islamia University of Bahawalpur, Bahawalpur, Pakistan \\ ${ }^{3}$ Department of Radiation Physics, University of Texas MD Anderson Cancer Center Houston Texas, USA \\ ${ }^{4}$ Department of radiation Physics, Women University A \& J Kashmir, Bagh, Pakistan
}

Received July 31, 2015; Revised October 04, 2015; Accepted October 08, 2015; Published Online November 06, 2015

\section{Original Article}

\begin{abstract}
Purpose: The purpose of this analysis was to investigate dose distribution of Three Dimensional Conformal Radiation Therapy (3DCRT), Intensity Modulated Radiation Therapy (IMRT) and Volumetric Modulated Arc Therapy (VMAT) for Head and Neck cancer using 3-dimensional PRESAGE® dosimeter. Method: Computer Tomography (CT) scans of Radiological Physics Center (RPC) Head and Neck anthropomorphic phantom with both RPC standard insert and PRESAGE® insert were acquired separated with Philipp's CT scanner and both CT scans were exported via DICOM to the pinnacle treatment planning system (TPS). Each plan was delivered twice to the RPC phantom first containing the RPC standard insert having Thermoluminescent detectors (TLD) and film dosimeters and then again containing the PRESAGE® insert having three dimensional dosimeter (PRESAGE®) by using a Varian True beam linear accelerator. After irradiation, the standard insert including point dose measurement (TLD) and planner GafChromic ${ }^{\circledR}$ EBT film measurement was read using RPC standard procedure. The 3D dose distribution from PRESAGE® was read out with the Duke Midsized optical scanner dedicated to RPC (DMOS-RPC). Dose volume histogram (DVH), mean and maximal doses for organ-at-risk (OARs) were calculated and compared among each Head and Neck technique. The prescription dose was same for all Head and Neck radiotherapy techniques which was 6.60 Gy per friction. Beam profile comparison and gamma analysis were used to quantify agreement among film measurement, PRESAGE® measurement and calculated dose distribution. Quality assurances of all plans were performed by using ArcCHECK method. Results: VMAT delivered the lowest mean and maximal doses to organ at risk (spinal cord and parotid) than IMRT and 3DCRT. Such dose distribution was verified by absolute dose distribution using TLD system. 2D gamma 5\%/3 mm criteria of Pinnacle vs. EBT2 film 3DCRT (92.34\%), IMRT (92.3\%) and VMAT (96.63\%) in axial plan respectively. It was also found that agreement between PRESAGE® and pinnacle along the axial, sagittal and coronal plans VMAT agreement was better than IMRT and 3DCRTplan excludes a $7 \mathrm{~mm}$ rim at the edge of the dosimeter using 2D gamma map criteria $( \pm 5 \% / 3 \mathrm{~mm})$ with $5 \%$ threshold dose. Profile showed good agreement for all plans between film, PRESAGE® and pinnacle. 3D gamma was performed for planning target volume (PTV) and organ at risks (OARs) VMAT and 3DCRT endow with better agreement than IMRT. Conclusion: VMAT delivered lowered mean and maximal doses to organ at risk and better PTV coverage. TLD, EBT film and PRESAGE® dosimeter has suggested that VMAT would be superior modality for the treatment of Head and Neck cancer than IMRT and 3DCRT.
\end{abstract}

Keywords: RPC; 3DCRT; IMRT; VMAT; EBT2 FILM; TLD; PRESAGE ${ }^{\circledR}$

\section{Introduction}

The requirement for accurate and quick practical three dimension dosimetry has become a fundamental part of the radiation delivery and complex treatment process. The ideal dosimeter exhibits properties like tissue equivalent. ${ }^{1}$ The scattering and absorption properties of the radiation dosimeter should be comparable to the water. ${ }^{2}$ The radiological properties and the formulation have been studies by many writers and it was observed that PRESAGE ${ }^{\circledR}$ (Heuris Inc., Skillman, NJ) has effective atomic number (7.6) close to water (7.42). ${ }^{3}$ PRESAGE $^{\circledR}$ is a radiochromic three dimensional dose measuring dosimeter which is composed of polyurethane and radiochromic components (Leuco dyes) and halogen containing free radical initiator ${ }^{4}$ that have 
an optical attenuation coefficient that changes linearly with absorbed dose. ${ }^{5}$ The arrangement of PRESAGE ${ }^{\circledR}$ and an optical CT scanner has addressed this require to measured the dose in three dimensional (3D)..$^{6-7}$

The Radiological Physics Center (RPC) head and neck credentialing analysis is usually done by hospital that want to participate in the IMRT head and neck clinical trial guideline of the Radiation Therapy Oncology Group (RTOG). ${ }^{8}$ This analysis covers an IMRT plan created by the participant institution or hospital with irradiation of RPC head and neck phantom to encounter dose limit defined in the RTOG guideline.

The TLD and EBT film were used to achieve required dosimetric test into the RPC head and neck phantom. If the measured dose is within $7 \% / 4 \mathrm{~mm}$ gamma criteria with calculated dose by Treatment Planning System (TPS) at a limited number of points then participating institution passes the credentialing analysis. It has been observed that $25 \%$ institution fail to achieve require credentialing criteria at first attempt, out of 342 irradiations. ${ }^{9}$ To solve such problem, an urgent requirement for accurate three dimensional dosimeter i.e. PRESAGE ${ }^{\circledR}$ has been introduced to maintain of IMRT quality assurance. ${ }^{10-11}$.

Many studies had been done on PRESAGE ${ }^{\circledR}$ dosimeter that shows acceptance agreement between measured doses to calculated reference doses. Newton et al. ${ }^{12}$ indicated that PRESAGE $^{\circledR}$ 3D measurements were comprehensive and efficient, because alignment errors are negligible, and all parameters for multiple fields could be obtained from a single dosimeter and scan. However, achieving accurate superficial data (within $4 \mathrm{~mm}$ ) is not yet feasible due to optical surface artifacts. PDD values at $2 \mathrm{~cm}$ depth varied from $\sim 72 \%$ for the $40 \mathrm{~mm}$ field, down to $\sim 55 \%$ for the 1 mm field. EBT and PRESAGE ${ }^{\circledR}$ PDDs agreed within $\sim 3 \%$ in the typical therapy region $(1-4 \mathrm{~cm})$. At deeper depths the EBT curves were slightly steeper $(2.5 \%$ at $5 \mathrm{~cm})$. These results indicate good overall consistency between ion-chamber, EBT2 and PRESAGE ${ }^{\circledR}$ measured OFs, PDDs, and profiles.

It has been reported by Oldham et al. ${ }^{7}$ that good agreement between PRESAGE® and Eclipse TPS distribution showed by dose profile. Two dimensional gamma maps showed $98 \%$ pass rate between all three techniques. The best agreement between PRESAGE ${ }^{\circledR}$ and EBT were observed in the central plane. Gama pass rate were $94 \%$ between normalized PRESAGE $^{\circledR}$ and Eclipse three dimensional distributions. Some studies presented that PRESAGE ${ }^{\circledR} /$ Optical CT system had excellent precision, accuracy, reproducibility and robustness 3D dosimetry. ${ }^{13-14}$ Recently, it is reported that ferrous xylenol-orange gel/optical-CT dosimetry system were used to demonstrate the feasibility of three dimensional dosimetry in RPC head and neck phantom. ${ }^{15}$
Previous work has focused on the fundamental dosimetric characteristics of PRESAGE ${ }^{\circledR}$ and investigation of the feasibility of the PRESAGE ${ }^{\circledR} /$ pptical CT system for 3D dosimetry. The aim of this study is to compare different Head and neck radiotherapy techniques such as 3DCRT, IMRT and VMAT by using PRESAGE ${ }^{\circledR}$ dosimeter with the help of RPC credentialing phantom. To our knowledge this dosimetric comparison is not done for these radiotherapy techniques and this is first study of anthropomorphic Head and Neck PRESAGE ${ }^{\circledR}$ dosimeter.

\section{Methods and Materials}

\section{The RPC Insert and Phantom}

The removable dosimetry insert and the RPC phantom picture is shown in Figure 1(A). The RPC standard insert contain three independent structures which are visible in $\mathrm{x}$-ray CT scan due to different densities. The names of designated structure are planning target volume and two OARs such as spinal cord and parotid. Insert also contain three pieces of film and eight TLDs. Position of axial film and TLDs can be seen in Figure 1(B) and other two film are placed such that film form a single sagittal slice, when insert is assembled. More ever, two TLDs are in the paroid superior and inferior, two are in spinal cord superior and inferior and four more are in PTV.

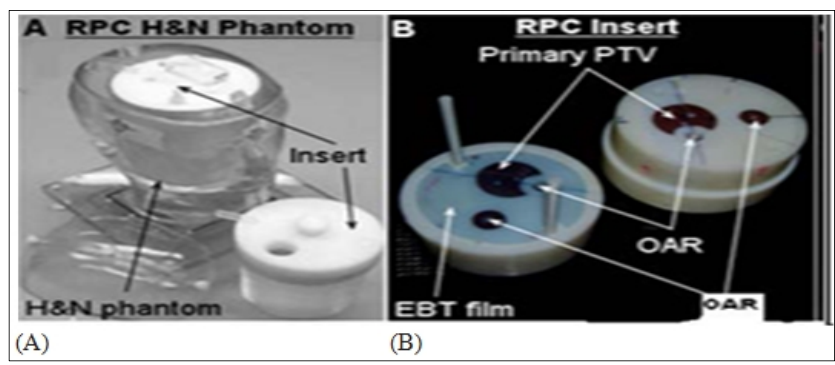

FIG. 1: (A) RPC Head and Neck an anthropomorphic Phantom; (B) Standard RPC Insert which containing PTV and OARs with dosimeter (EBT2, TLD).

\section{Treatment planning of RPC standard insert}

The RPC credentialing assessment comprises radiation of an anthropomorphic Head and Neck phantom containing the standard RPC insert with all treatment plans and comparing the measured dose with the planned dose. ${ }^{8}$ The RPC phantom was taken through the entire treatment planning procedure by medical physicists as an actual patient. All treatment plans were designed such that PTV coverage was $90 \%$ at least of the prescription dose for each technique and got best plan to spare OARs.

The RPC instruction was used to maintain room temperature and RPC head and neck phantom was filled with water. When there were no air bubbles in water phantom then insert was carefully placed inside phantom. To ensure 
consistent positioning during imaging and treatment, coplanar surface marker were placed on the outside of the phantom.

An x-ray CT scan was taken using a Philips CT scanner (Philips Healthcare, Andover, MA) with a slice thickness of $1.5 \mathrm{~mm}$ to ensure that the TLDs could be seen. The CT scan was imported into the Pinnacle 9.4 version treatment planning system, where the structures (PTV, OARs) and TLDs were manually contoured for all 3DCRT, IMRT and VMAT plans. Treatment planning was done using VMAT (four arc: $182^{\circ}-178^{\circ}, 180^{\circ}-184^{\circ}, 182 \mathrm{o}-178^{\circ}, 180 \mathrm{o}-184^{\circ}$ ), Nine field IMRT (beam angles: $200^{\circ}, 240^{\circ}, 280^{\circ}, 320^{\circ}, 0^{\circ}, 40^{\circ}, 80^{\circ}$, $120^{\circ}$ and $160^{\circ}$ ) has been commonly used at MD Anderson Cancer center, Houston and Four fields for 3DCRT (AP, PA, Lt Lat and Rt Lat) were used. True Beam linear accelerator was used for dose delivery with photon of 6MV energy and C.C convolution algorithm was used for dose calculation. Whereas adaptive convolve algorithm was used for optimization for both IMRT and VMAT.

\section{Quality assurance, TLD and EBT film analysis}

Earlier to treatment, routine ArcCHECK (Sun Nuclear, Melbourne, FL) QA procedures used at MD Anderson were followed to check consistency of planned fluence with delivered fluence for individual beams for all plans (3DCRT, IMRT and VMAT). After ArcCHECK QA, the phantom was transferred to the couch of a Varian True beam (Varian Medical Systems, Palo Alto, CA), where the treatment was delivered to the phantom. The treatment plans including the dose calculation and insert were sent to RPC for dose measurement and analysis. The RPC's standard procedures were used to read the irradiated thermoluminescent dosimeters and EBT2 films to decrease ambiguity. An independent dose distribution authentication was performed using EBT2 film (ISP Corp, Wayne, NJ, USA). The basic reasons to use EBT2 film are its temporal stability, directional independence and easy for self development. ${ }^{16}$

The delivered absolute dose to the mean computed dose values inside the TLD contours were used to compare the measured thermoluminescent dosimeter doses. ${ }^{13}$ In our analysis, the irradiated films were sampled at a $0.3 \mathrm{~mm} \times 0.3$ mm pixel resolution and compared with the related treatment plan dose distribution. The consequence of lower resolution in film is acceptable and observed as sharper. A set of three tiny pinholes in each film were used to register the film measurements and planned dose distributions within the RPC head and neck phantom. At RPC, the recorded dose in the TLDs and the EBT film (henceforth called EBT dose) were compared with the planned dose. ${ }^{8}$ A calibration curve was prepared at the time of experimental irradiation as shown in Figure 2. The films were digitized using a 48-bit transmission/reflection flatbed photo scanner (Flat-Bed Epson-10000XL). Each film was scanned in transmission mode of three channels red, green and blue but only the red channel was extracted for analysis because EBT has a maximum response to red light at $633 \mathrm{~nm} .{ }^{15} \mathrm{We}$ did gamma analysis with RPC film software and film dose were scaled with TLD dose. The calibration curve was applied to the EBT film to convert (OD) to dose.

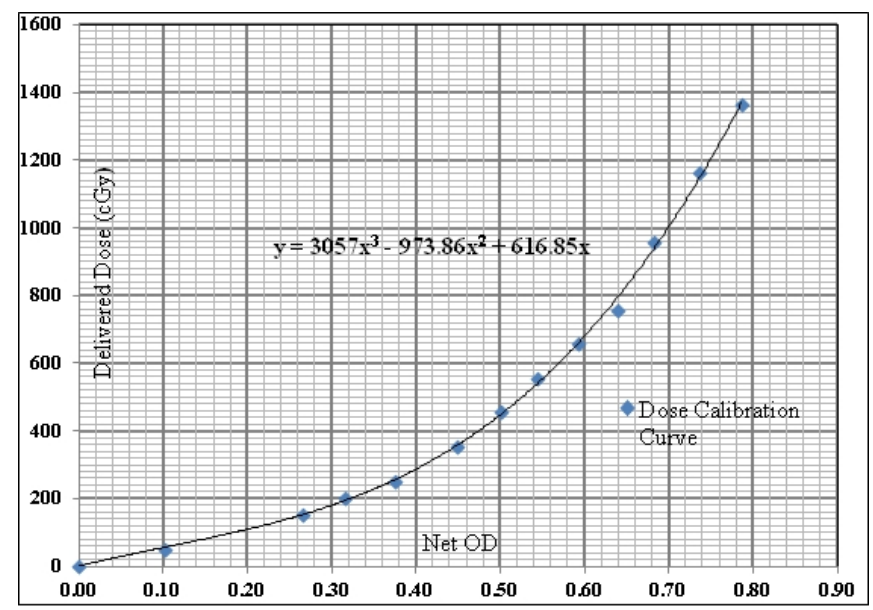

FIG. 2: Calibration curve used to convert independent 2D dose distribution verification was made using RPC GAFCHROMIC EBT2 film scanner.

\section{D dose measurement using PRESAGE ${ }^{\circledR}$ /optical-CT}

The standard insert was removed and replaced with a modified insert containing a PRESAGE ${ }^{\circledR} 3 \mathrm{D}$ dosimeter with no disturbing the phantom alignment on the treatment machine to reduce the setup errors. Treatment planning and treatment delivery to the phantom with PRESAGE ${ }^{\circledR}$ dosimetry insert was similar to that of the standard RPC insert with only minor differences. Optical-CT was used for $3 \mathrm{D}$ dose readout from the irradiated PRESAGE ${ }^{\circledR}$ dosimeter. A solid, radiochromic leuco dye doped polyurethane plastic PRESAGE ${ }^{\circledR}$ dosimeter Heuris Inc., Skillman, NJ) was molded to fit inside a plastic sleeve that was compatible with RPC Head and Neck phantom as shown in Figure 3. The PRESAGE ${ }^{\circledR}$ dosimeter along with the compatible sleeve is hence onward called the PRESAGE ${ }^{\circledR}$ insert. Radiochromic properties of PRESAGE $^{\circledR}$ have been well characterized. ${ }^{2}$ The formulation used in this study had an effective $\mathrm{Z}$ number of 7.6 and a physical density of $1.07 \mathrm{~g} / \mathrm{cm}^{3} .5,3$

The radiochromic response was determined spectrophotometrically using cuvette irradiations and was found to be linear with sensitivity of 0.046 optical density (OD) changes per $\mathrm{cm}$ per Gy. Using the cuvette sensitivity data, it was estimated that a dose of 4 Gy would result in an OD change. The treatment planning and delivery for the phantom with PRESAGE $^{\circledR}$ insert was similar to the procedures for an actual patient. A CT scan of the phantom with PRESAGE ${ }^{\circledR}$ insert was acquired by swapping with the RPC insert. The surface markers already present on the phantom were used to position the phantom in the same orientation as that for the CT scan with RPC insert. The CT 
scan of the phantom with the PRESAGE ${ }^{\circledR}$ insert was imported into the Pinnacle TPS and registered with the CT scan of the phantom with the RPC insert using the auto register tool, with the only exception that the prescription dose was reduced from 6.6 to $4 \mathrm{~Gy} /$ fraction. This was required because of the dose limit placed by the OD change requirement of PRESAGE ${ }^{\circledR}$. A dose of 6.6 Gy would have induced a suboptimal OD change resulting in undesirable artifacts for this particular formulation of PRESAGE ${ }^{\circledR} .17$

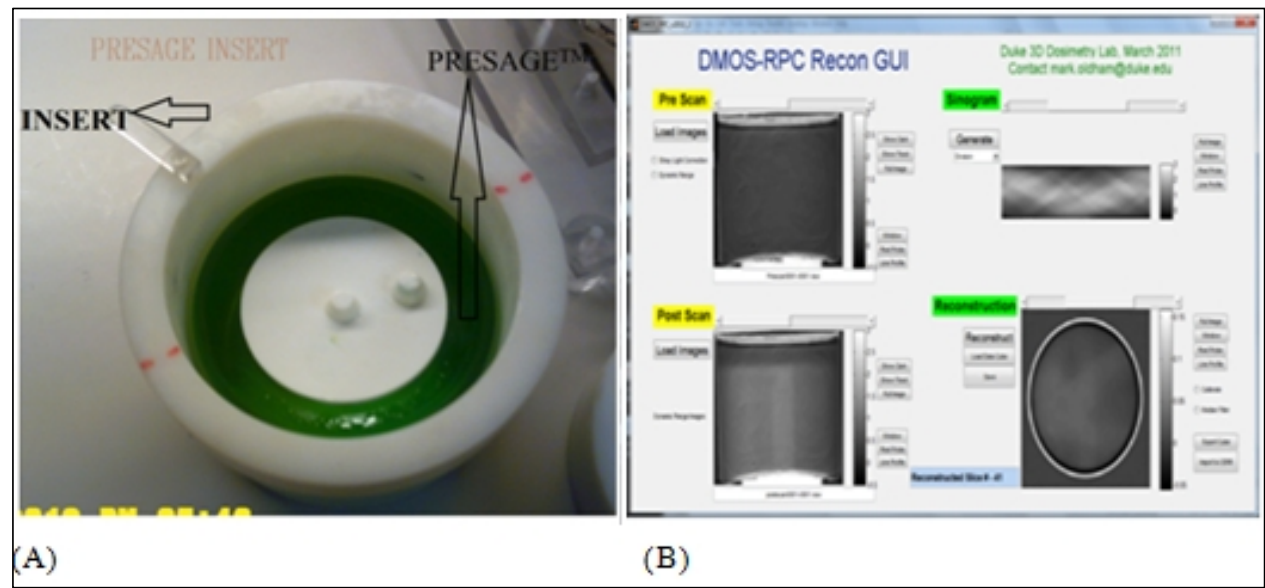

FIG. 3: (A) PRESAGE® Insert containing plastic Insert and PRESAGE ${ }^{\circledR}$; (B) DMOS reconstruction graphical user interfaces. Upper left quadrant display projection images associated with the pre-irradiation scan and the lower left quadrant display projection images of the post-irradiation scan. The Dosimeter was irradiated with nine field IMRT plan.

Before treatment, ArcCHECK QA procedures at MD Anderson were followed to verify consistency of planned fluence and delivered fluence for each beam. After all plans QA i.e 3DCRT, IMRT and VMAT the phantom with PRESAGE $^{\circledR}$ insert was aligned on the treatment couch using surface markers and the new plans with $4 \mathrm{~Gy}$ prescription dose was delivered. A concern with scaling the prescription dose was that it might cause differences in relative fluence, MLC leaf motion, and relative dose distribution. To evaluate the significance of changing prescription dose on the consistency of the relative fluence between the two plans, the ArcCHECK QA of all three plans measurements from the six plans were analyzed. In addition, the Dynamic MLC log files that were generated after delivery of each field were analyzed to independently ascertain the relative consistency of MLC leaf motion. After the treatment all plans, the PRESAGE ${ }^{\circledR}$ dosimeter was kept refrigerated at $4^{\circ} \mathrm{C}$ and away from room light for 12 hour then the 3D dose distribution was read out using optical-CT scanner with the Duke Midsized optical scanner (DMOS) dedicated for the RPC (DMOS-RPC) [Duke University, Durham, NC] using 1 degree per step to produce 360 projection images. The DMOS-RPC scanner consists of matched telecentric source and image lenses that provide a fixed field of view (FOV). The DMOS was intended to use with PRESAGE ${ }^{\circledR}$ to find out the linear change in optical density against the ionizing radiation. The DMOS-RPC scanner reduces optical refraction artifacts by surrounding the PRESAGE $^{\circledR}$ dosimeters within a refractive index matching fluid. The fluid (mixture of octyl salicylate and octyl methoxy cinnamate) was filtered before optical-CT scanning to remove suspended impurities. The PRESAGE ${ }^{\circledR}$ dosimeters were scanned before and after irradiation. The scanner was configured for a voxel size of $1 \mathrm{~mm}^{3}$ comprised a linear projection scan.

\section{Data registration and dose analysis}

The images from DMOS and Pinnacle TPS were exported to the CERR (Washington University, St Louis, MO) a MATLAB based software. Three types of data were available for analyses of each plan: (1) Pinnacle calculated dose distribution (2) 2D dose distribution of GAFCHROMIC EBT2 film (3) PRESAGE ${ }^{\circledR}$ dosimeter dose distribution for each plans i.e. 3DCRT, IMRT and VMAT. The calculated dose distribution from TPS (Pinnacle) was compared to measured dose distribution from PRESAGE ${ }^{\circledR}$ and GafChromic EBT2 for each plan with correspondence plans then again were compared among each techniques of Head and Neck radiotherapy. GafChromic EBT2 films doses were measured by using RPC images software. Line profiles and Gamma analysis were used to quantify the agreement between calculated and measured dose distribution. Whereas 3D gamma map were used between PRESAGE ${ }^{\circledR}$ dose distribution and Pinnacle dose distribution in the specific region of interest (ROI) i.e. PTV, spinal cord and parotid. The gamma map used criteria of $5 \% / 3 \mathrm{~mm}$ dose difference /distance to agreement with maximum $5 \%$ threshold dose, which was more stringent than currently used for RPC Head and Neck credentialing protocols $(7 \% / 4 \mathrm{~mm})$.

\section{Results and Discussion}

Earlier to a straight comparison of $\operatorname{PRESAGE}^{\circledR}$ with the 
gold-standard EBT film, it was compulsory to make obvious that the relative dose distributions were identical in the standard RPC insert (containing EBT) and the PRESAGE ${ }^{\circledR}$ insert. Differences could arise from the scaling of the dose prescription between these two plans (6.6 Gy for EBT and 4 Gy for the PRESAGE ${ }^{\circledR}$ ) and the differences in CT numbers between the PRESAGE ${ }^{\circledR}$ and EBT inserts.
Comparison of relative fluence maps measured by ArcCHECK QA device showed that the relative fluences were essentially identical between all these plans. With an extremely high tolerance criterion of 3\% dose difference and $3 \mathrm{~mm}$ DTA, a greater than $98 \%$ gamma pass rate was achieved for all plans as shown in Figure 4.

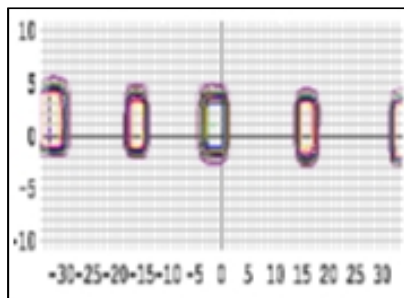

(A)

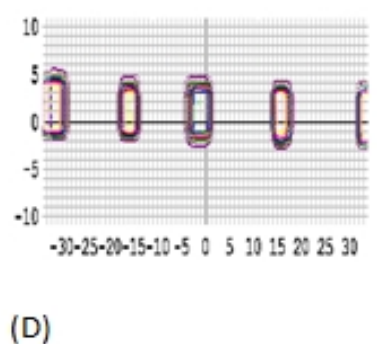

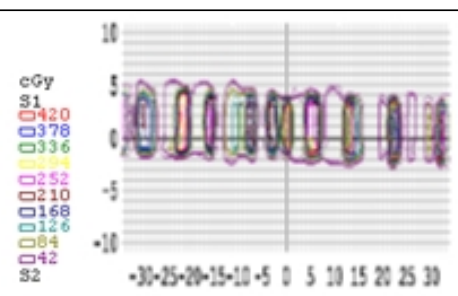

(B)

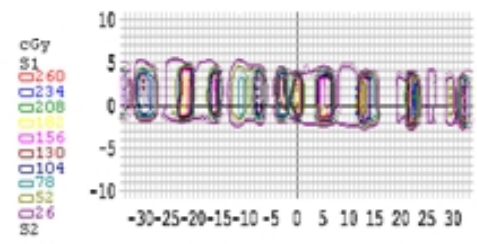

(E)

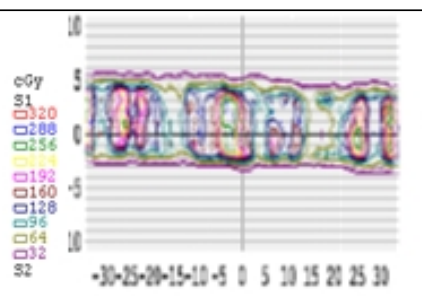

(C)

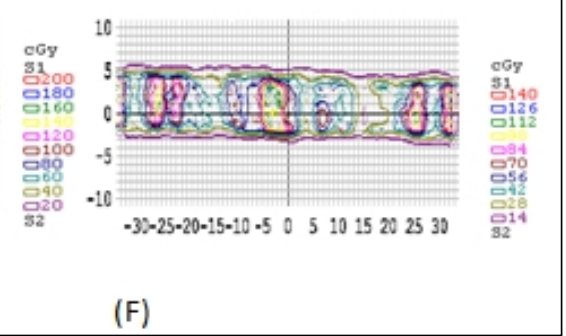

FIG. 4: ArcCHECK Quality assurance (Q.A.) of 3DCRT (A), IMRT (B) and VMAT (C) plans with RPC standard insert and (D), (E) and (F) show QA. of 3DCRT, IMRT and VMAT respectively with PRESAGE ${ }^{\circledR}$ insert.
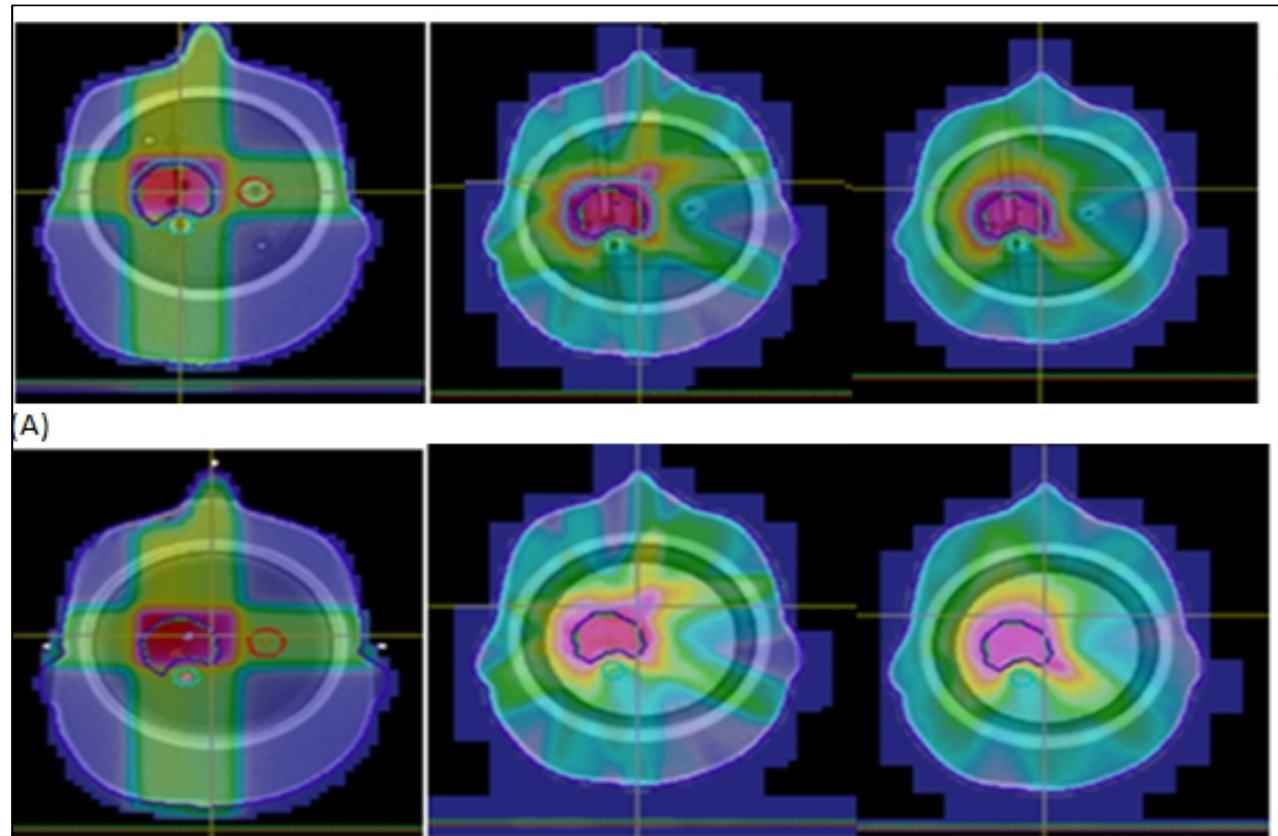

B)

FIG. 5: (A) Pinnacle ${ }^{\circledR}$ version 9.4 (TPS) dose distribution with dose prescription 6.6Gy in axial views of different plans 3DCRT, IMRT and VMAT from left to right respectively with RPC standard insert; (B) Pinnacle ${ }^{\circledR}$ version 9.4 (TPS) dose distribution with dose prescription 4Gy in axial views of different plans 3DCRT, IMRT and VMAT from left to right respectively with PRESAGE® insert scan. 
The comparison of relative dose distributions from the two treatments plans (6.6 Gy and $4 \mathrm{~Gy}$ ), as calculated by the Pinnacle TPS are shown in Figure 5 for different Head and Neck radiotherapy techniques. These represented isodose lines in color wash are extracted from CERR. It is explored dose distribution does not change by reducing the prescription dose from $6.6 \mathrm{~Gy}$ to $4 \mathrm{~Gy}$ and also will agreed with Sakhalkar et al. ${ }^{13}$ results.

\section{Intercomparison of 3DCRT, IMRT and VMAT plans using line profiles and gamma criteria among PRESAGE $^{\circledR}$ dose, EBT dose, and pinnacle calculation}

\section{Isodose line profile}

Figure 6 illustrates the isodose line profile comparison taken along the anterior-posterior of PTV for 3DCRT, IMRT and VMAT plans between Pinnacle TPS with EBT 5(A, B, C) and PRESAGE $^{\circledR} 5(\mathrm{D}, \mathrm{E}, \mathrm{F})$. The Pinnacle dose distribution is smoother with less noise than either of the measured dose distribution as expected. Various comparatively minor differences can be discerned between the distributions due to setup error but trends are not readily apparent and it is not possible to state whether the Pinnacle dose distribution agrees more closely with one or the other of the measured distributions. In some cases both measured distribution appear to show a discrepancy with the Pinnacle dose distribution. An example is the line profiles at the edges of graph as shown in Figure 6. In general, consistent or systematic trends were discerned and the distributions appear very similar with discrepancies attributed to be within the nose limit. Another consideration is that the two measured distribution EBT and PRESAGE ${ }^{\circledR}$ actually correspond to two independent deliveries of the same plans. Any variation in the mechanism of deliveries would also contribute to difference in the measured distribution.

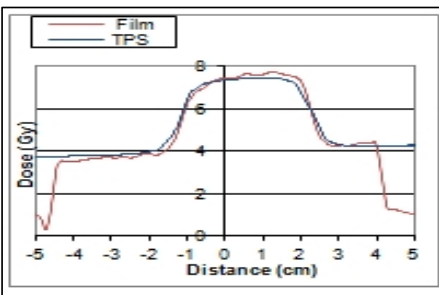

(A)

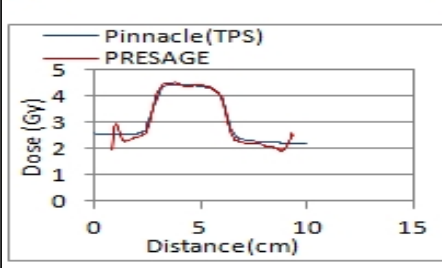

(D)

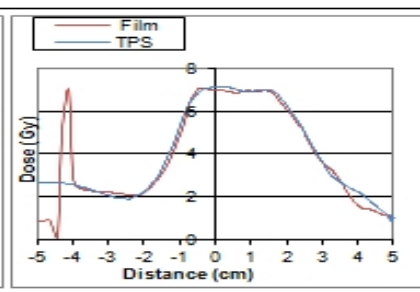

(B)

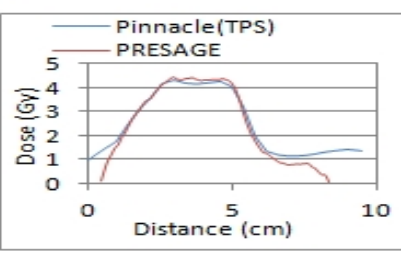

(E)

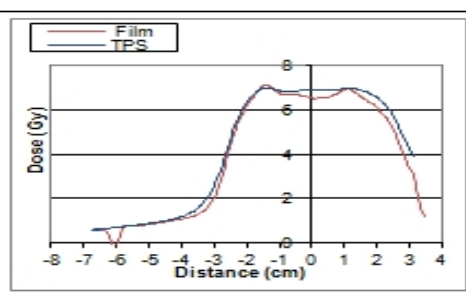

(C)

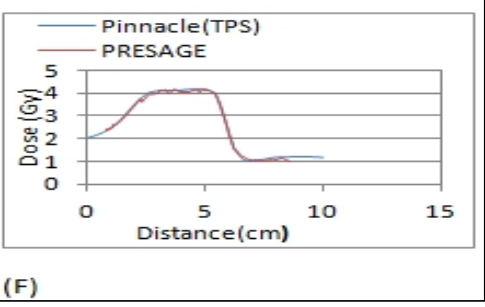

FIG. 6: Comparison of dose profiles for Pinnacle (TPS) and film measurement (A, B, C), Pinnacle (TPS) and PRESAGE® (D, E, F) for 3DCRT, IMRT and VMAT plan along Ant-Post profiles respectively.

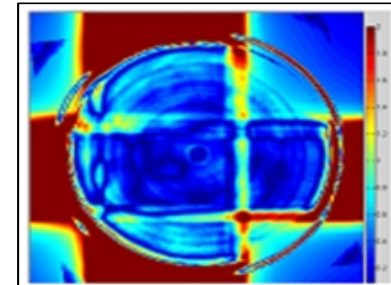

(A)

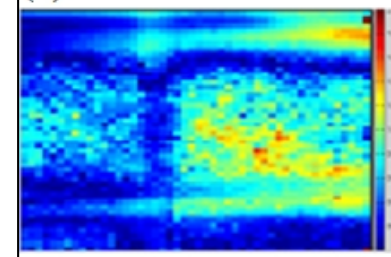

(B)
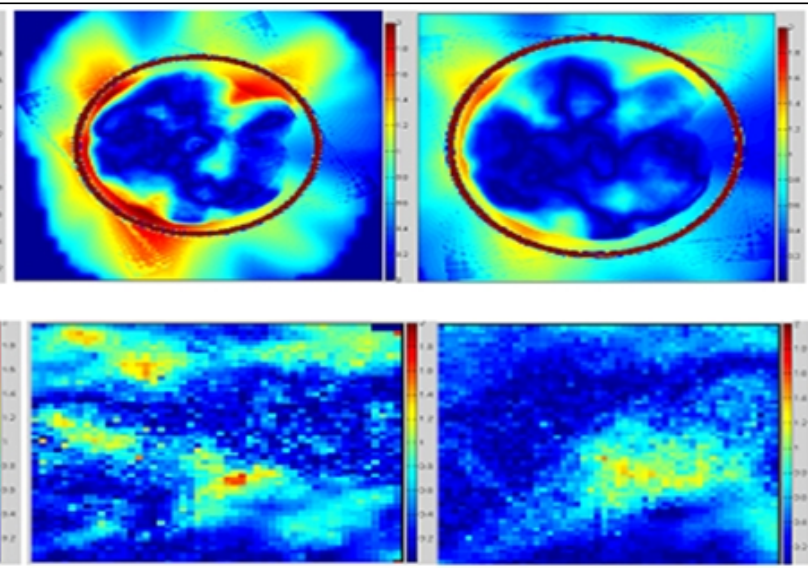

FIG 7: (A) 2D Gamma 5\%/3mm criteria of Pinnacle vs. PRESAGE® 3DCRT (96.35\%), IMRT (96.65\%) and VMAT (99.82\%) in axial plan respectively from left to right with 5\% threshold dose through PTV; (B) 2D Gamma 5\%/3mm criteria of Pinnacle vs. EBT2 film 3DCRT (92.34\%), IMRT (92.3\%) and VMAT (96.63\%) in axial plan respectively from left to right. 
Comparison of normalized 2D dose maps and dose profiles from PRESAGE ${ }^{\circledR}, E B T$, and Pinnacle distributions in the central axial plane is shown in Figure 6. Dose profile comparisons showed close agreement of PRESAGE ${ }^{\circledR}$ with Pinnacle. The agreement was good even in the dose-gradient regions between the PTV and OARs, which can be key sources of dosimetric errors. This difference represents an uncertainty arising from the present calibration procedure. The PRESAGE ${ }^{\circledR}$ dose in the experimental large-volume insert was determined using the calibration curve obtained from the irradiation of small volume cuvettes using true beam machine. Part of the difference may be due to a volume sensitivity effect which needs further investigation. In addition, part of the difference might be attributed to the different sensitivities of the spectrophotometer used to measure the OD for cuvette calibration and the Duke RPC scanner used to measure the OD in the much larger experimental insert. ${ }^{19}$ In general, all beam profiles of VMAT look better agreement then IMRT and 3DCRT plans.

\section{Gamma Map comparison}

Gamma map investigation was used for further broad authentication of the extent of agreement between Pinnacle,
EBT and PRESAGE ${ }^{\circledR}$ as shown in Figure 7. Gamma criteria of $5 \%$ dose difference and $3 \mathrm{~mm}$ DTA were used, substantially less than the $7 \%, 4 \mathrm{~mm}$ criteria used in the RPC credentialing test. A 2D gamma comparison between PRESAGE $^{\circledR}$ and the Pinnacle TPS distribution Figure 7(A) yielded a high pass rate of $98 \%$ this number excludes a $5 \mathrm{~mm}$ rim at the edge of the dosimeter, where edge artifacts occur. 2D gamma results were 96.35\% (axial), 98.74\% (sagittal), $97.76 \%$ (coronal) for 3DCRT, 95.23\% (axial), 99.66\% (sagittal), 98.06\% (coronal) for IMRT, 99.82\% (axial) and 99.78\% (sagittal), 98.38\% (coronal) for VMAT plans between PRESAGE ${ }^{\circledR}$ and pinnacle with 5\% threshold dose through PTV.

3D gamma showed excellent results in 3D volume of region of interest (PTV and OARs) of all three plans between PRESAGE $^{\circledR}$ and Pinnacle as shown in Table 1. VMAT illustrated better gamma results than the other two techniques. The routine credentialing analysis comparing Pinnacle with EBT as shown in Figure 7(B) was confirming RPC credentialing criteria of $7 \% / 4 \mathrm{~mm}$. The disappointment at the edge is caused by an edge artifact in the PRESAGE ${ }^{\circledR}$ measurement. ${ }^{13}$

TABLE 1: 3D Gamma results with criteria of 5\%/3mm along 5\% threshold dose passing (Voxel\%) Pinnacle (TPS) and PRESAGE ${ }^{\circledR}$.

\begin{tabular}{lccc}
\hline \hline ROI & VMAT (Voxel\%) & IMRT (Voxel\%) & 3DCRT (Voxel\%) \\
\hline PTV & 98.14 & 92.1 & 96.93 \\
Spinal Cord & 99.9 & 85.3 & 99.9 \\
Parotid & 99.5 & 96.19 & 98.74 \\
\hline \hline
\end{tabular}

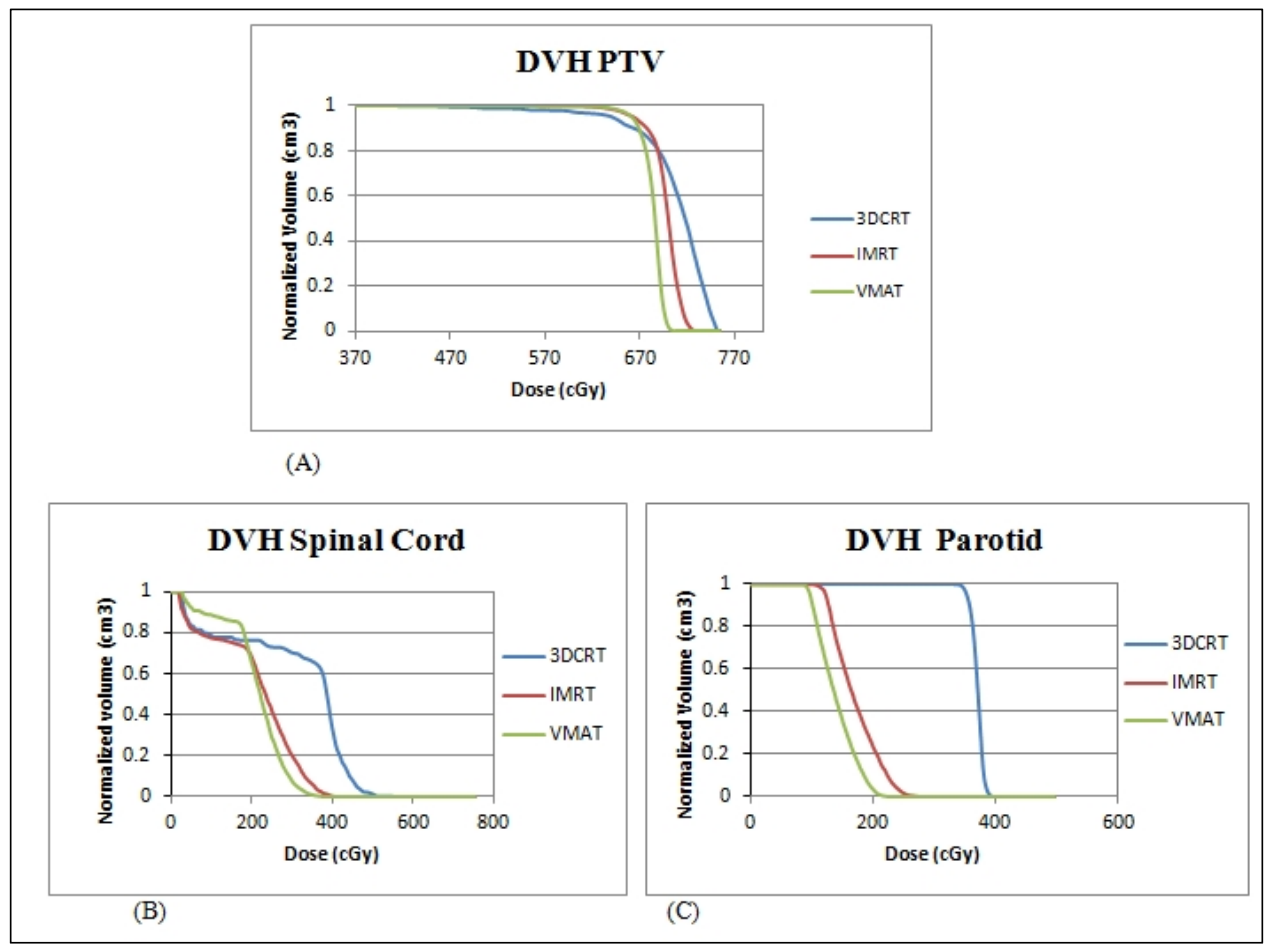

FIG. 8: Cumulative DVH for PTV (A), spinal cord (B) and Simulated Parotid Gland (C) for 3DCR., IMRT and VMAT. 
Dose volume histogram (DVH) comparison among different Head and Neck radiotherapy techniques

DVH provide dose volume information for the organs contoured in the treatment planning system. Figure 8 showed the dose volume contribution to the PTV from the different Head and Neck radiotherapy techniques. During treatment planning, it was made sure that prescription dose was delivered at marked isocenter and PTV coverage was calculated for each technique. PTV coverage was $90.80 \%$, $95.80 \%$ and $95.82 \%$, for 3DCRT, IMRT and VMAT, respectively as shown in Figure 8(A), which reveals that VMAT target coverage were better than other Head and Neck techniques (IMRT and 3DCRT).

TABLE 2: Maximum and Mean doses for Organs-at-Risk (OARs) for 3DCRT. IMRT and VMAT

\begin{tabular}{ll|c|c}
\hline \hline \multicolumn{2}{l|}{ Techniques } & Parotid & Spinal Cord \\
\hline 3DCRT & Measn dose (Gy) & 3.7 & 3.1 \\
& Max. dose (Gy) & 4 & 5.6 \\
IMRT & Measn dose (Gy) & 1.7 & 2.1 \\
& Max. dose (Gy) & 2.9 & 4.2 \\
\hdashline VMAT & Measn dose (Gy) & 1.4 & 2.1 \\
& Max. dose (Gy) & 2.3 & 4 \\
\hline \hline
\end{tabular}

VMAT delivered the lowest maximal doses than all other studied techniques to spinal cord $(4.0 \mathrm{~Gy})$ and parotid (2.3 Gy) as shown in Figure 8(B and C). In comparison, maximal doses for 3DCRT were $40 \%$ and $74 \%$ higher than VMAT for spinal cord and parotid, respectively. Whereas, IMRT delivered maximal 5\% and 26\% higher doses at spinal cord and parotid than VMAT as shown in Table 2.

\section{TLD results}

TLD results passed the RPC 7\% dose difference criteria, i.e., the measured dose agreed well with dose calculations within the $7 \%$ dose difference. Results showed that the TLD dose measurements in PTV were within 7\% of the Pinnacle calculations for all Head and Neck radiotherapy treatment modalities. In addition to TLD dose measurement, EBT dose measurement scaled to the TLD dose was used for relative $2 \mathrm{D}$ dosimetric verification in the central axial plane. TLD results showed that VMAT plans delivered lower doses to OARs as compared to other IMRT and 3DCRT plans and each plan was delivered three times for the reproducibility as shown in Figure 9.

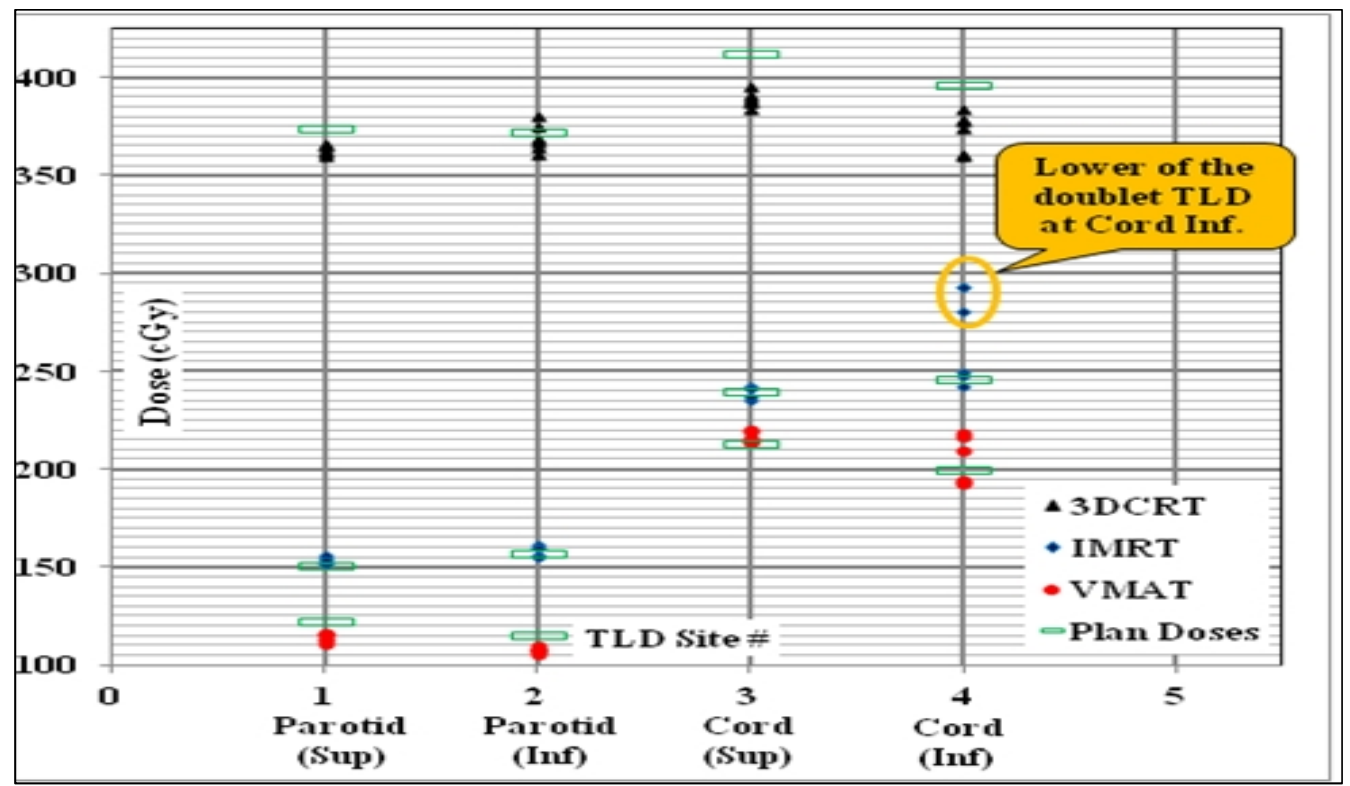

FIG. 9: TLD [Site1-2(Parotid sup-inf), 3-4(spinal Cord sup-inf)] Doses to organ at risk during head and neck radiotherapy techniques (3DCRT, IMRT, VMAT).

\section{Conclusion}

VMAT delivered lowered mean and maximal doses to OARs with better PTV coverage during Head and Neck radiotherapy. This work demonstrates the dosimetric comparison and the implementation of the PRESAGE ${ }^{\circledR}$ /optical-CT 3D dosimetry system in the RPC Head and Neck phantom using different Head and Neck radiotherapy treatment modalities i.e. 3DCRT, IMRT and VMAT. The PRESAGE $^{\circledR}$ measurement was hence used as a 3D standard to compare the accuracy of Pinnacle 3D dose calculations. The Pinnacle dose calculations showed good agreement (99\% pass rate) with gamma criteria of $5 \%$ dose difference and 3 mm DTA with the PRESAGE ${ }^{\circledR}$ measurement at region of interest (ROI) 3D volume (PTV and OARs) for VMAT and 3DCRT plans and $95 \%$ pass rate for complex nine field IMRT plan too. TLD, EBT film and PRESAGE ${ }^{\circledR}$ dosimeters suggest that VMAT is better for the treatment of Head and Neck cancer than IMRT and 3DCRT. 


\section{Conflict of interest}

The authors declare that they have no conflicts of interest. The authors alone are responsible for the content and writing of the paper.

\section{Acknowlegments}

Authors are thankful to the Higher Education Commission (HEC), Pakistan for providing a scholarship for Mr. Jalil ur Rehman. The authors also thank Mr. Pei Fong (Senior Medical Physicist) from radiation Physics at The University of Texas MD Anderson Cancer Center for help in treatment planning and valuable discussions.

\section{References}

1. Baldock C, De Deene Y, Doran S, et al. Polymer gel dosimetry. Phys Med Biol. 2010;55:R1-63.

2. Sakhalkar H, Sterling D, Adamovics J, et al. Investigation of the feasibility of relative $3 \mathrm{D}$ dosimetry in the Radiologic Physics Center Head and Neck IMRT phantom using presage/optical-CT. Med Phys. 2009;36:3371-7.

3. Sakhalkar HS, Adamovics J, Ibbott G, Oldham M. A comprehensive evaluation of the PRESAGE/optical-CT 3D dosimetry system. Med Phys. 2009;36:71-82.

4. Doran SJ. The history and principles of chemical dosimetry for 3D radiation fields: Gels polymers and plastics. Appl Radiat Isot. 2009; 67: 393-8.

5. Brown S, Venning A, De Deene Y, et al. Radiological properties of the PRESAGE and PAGAT polymer dosimeters. Appl Radiat Isot. 2008;66:1970-4.

6. Guo P, Adamovics J, Oldham M. A practical three-dimensional dosimetry system for radiation therapy. Med Phys. 2006;33:3962-72.

7. Oldham M, Sakhalkar H, Guo P, Adamovics J. An investigation of the accuracy of an IMRT dose distribution using two- and three-dimensional dosimetry techniques. Med Phys. 2008;35:2072-80.

8. Molineu A, Followill DS, Balter PA, et al. Design and implementation of an anthropomorphic quality assurance phantom for intensity-modulated radiation therapy for the Radiation Therapy Oncology Group. Int J Radiat Oncol Biol Phys. 2005;63:577-83.

9. Ibbott GS, Followill DS, Molineu HA, et al. Challenges in credentialing institutions and participants in advanced technology multi-institutional clinical trials. Int J Radiat Oncol Biol Phys. 2008;71:S71-5.

10. Babic S, Battista J, and Jordan K. Three-dimensional dose verification for intensity-modulated radiation therapy in the radiological physics centre head-and-neck phantom using optical computed tomography scans of ferrous xylenol-orange gel dosimeters. Int J Radiat Oncol Biol Phys. 2008; 70:1281-91.

11. McJury M, Oldham M, Cosgrove VP, et al. Radiation dosimetry using polymer gels: methods and applications. Br J Radiol. 2000;73:919-29.

12. Newton J, Oldham M, Thomas A, et al. Commissioning a small-field biological irradiator using point, 2D, and 3D dosimetry techniques. Med Phys. 2011;38:6754-62.

13. Sakhalkar HS, Adamovics J, Ibbott G, Oldham M. An Investigation into the Robustness of Optical-CT Dosimetry of a Radiochromic Dosimeter Compatible with the RPC Head-and-Neck Phantom. J Phys Conf Ser. 2009;164:12059.

14. Sakhalkar H, Oldham M. Fast high-resolution 3D dosimetry utilizing a novel optical-CT scanner incorporating tertiary telemetric collimation. Med Phys. 2008;35:101-11.

15. Devic S, Seuntjens J, Sham E, et al. Precise radiochromic film dosimetry using a flat-bed document scanner. Med Phys 2005;32:2245-53.

16. Babic S, Battista J, and Jordan K. Three-dimensional dose verification for intensity-modulated radiation therapy in the radiological physics centre head-and-neck phantom using optical computed tomography scans of ferrous xylenol-orange gel dosimeters. Int J Radiat Oncol Biol Phys. 2008; 70: 1281-91.

17. Oldham M, Kim L. Optical-CT gel-dosimetry. II: Optical artifacts and geometrical distortion. Med Phys. 2004; 31: 1093-104. 